\title{
Male Partners of Infertile Couples with Seminal Infections of Human Papillomavirus Have Impaired Fertility Parameters
}

\author{
Edilson Damke, ${ }^{1}$ Fábio A. Kurscheidt, ${ }^{1,2}$ Valério A. Balani, ${ }^{2}$ Karen I. Takeda, ${ }^{2}$ \\ Mary M. T. Irie, ${ }^{1}$ Fabrícia Gimenes, ${ }^{1}$ and Marcia E. L. Consolaro ${ }^{1}$ \\ ${ }^{1}$ Clinical Cytology and STD Laboratory, Department of Clinical Analysis and Biomedicine, State University of Maringá, \\ Maringá, PR, Brazil \\ ${ }^{2}$ Sperm Analysis Section, São Camilo Laboratory, Maringá, PR, Brazil
}

Correspondence should be addressed to Marcia E. L. Consolaro; melconsolaro@gmail.com

Received 21 February 2017; Revised 11 June 2017; Accepted 2 July 2017; Published 1 August 2017

Academic Editor: Esteban Martinez

Copyright (C) 2017 Edilson Damke et al. This is an open access article distributed under the Creative Commons Attribution License, which permits unrestricted use, distribution, and reproduction in any medium, provided the original work is properly cited.

\begin{abstract}
Several studies have addressed the impact of viral infections on male infertility. However, it is still unknown whether human papillomavirus (HPV) can alter seminal parameters. The aim of this study was to determine the prevalence of HPV in the semen of male partners of couples seeking fertility evaluation. Additionally, we assessed the possibility that HPV infections affect seminal parameters. A total of 229 semen samples were collected from men in the Sperm Analysis Section of São Camilo Laboratory of Maringá, Brazil, between October 2015 and March 2016. Basic seminal parameters were analyzed, and HPV was detected and genotyped by polymerase chain reaction. HPV DNA was detected in $16.6 \%$ of samples. Of these, $10.5 \%$ had single type HPV infections, 6.1\% had multiple HPV infections, 5.7\% had exclusively high-risk HPV, and $6.1 \%$ had exclusively low-risk HPV. Samples positive for single and multiple types of HPV were associated with abnormal viscosity, and samples positive for multiple HPV types were also associated with hypospermia, higher $\mathrm{pH}$, and increased leukocyte numbers. These findings suggest that the male partners of infertile couples with seminal HPV infections may have prostate disturbances indicative of glandular dysfunction, which may influence fertility.
\end{abstract}

\section{Introduction}

Human papillomavirus (HPV) is one of the most common viral sexually transmitted diseases in both males and females worldwide [1]. Low-risk types of HPV (LR HPV) can cause genital warts, whereas persistent infection with high-risk types of HPV (HR HPV) is associated with anogenital and oropharyngeal cancers. In males infected with HPV, the virus can be found not only in the anal region, perineum, scrotum, glans, penile shaft, and urethra $[2,3]$ but also in the reproductive system (testis, epididymis, and ductus deferens) $[4,5]$. Moreover, the presence of HPV in semen has been documented previously [6-8]. However, it is still unknown whether HPV infection can affect semen fertility parameters.

Once it became established that the virus is primarily transmitted through direct epithelial contact [8], little attention was paid to the presence of HPV in semen.
However, HPV DNA was detected in $10 \%$ of semen samples from asymptomatic, sexually active young adult men [5], and Kaspersen et al. [8] found that $16.0 \%$ of semen from sperm donors contained HPV. HR HPV and multiple HPV infections were detected in $61.9 \%$ and $5.3 \%$ of these individuals, respectively. Additionally, HPV infection of the penile epithelium was found to be associated with the presence of HPV in semen, suggesting that seminal HPV might result from exfoliation of HPV-infected penile keratinocytes [9]. As a result, semen samples have high diagnostic value for the assessment of HPV infection in asymptomatic males [10].

So far, conflicting results have been reported on the effects of HPV infection on semen quality [9, 11-17]. Most previous studies on this topic were performed on relatively small cohorts [16-18]. Expanding knowledge in this area could provide valuable insight for guidelines on whether HPV testing is necessary in the diagnostic work-up for subfertile 


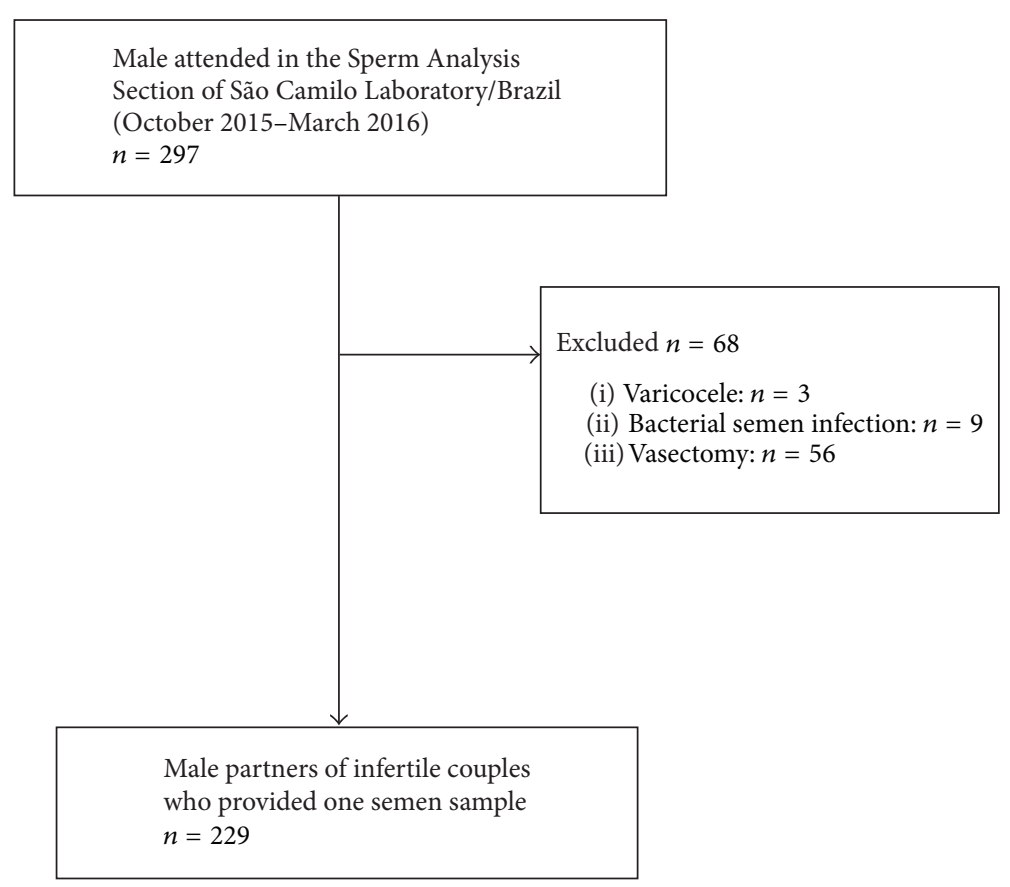

FIGURE 1: Flow chart of the study.

couples and in assisted reproduction procedures $[9,17]$. The aim of this study was to determine the prevalence of HPV in the semen of randomized male partners of couples seeking fertility evaluation. In addition, the possibility that HPV infection could affect seminal parameters, and thus fertility, was assessed.

\section{Materials and Methods}

2.1. Study Design and Approval. From October 2015 until March 2016, 297 males were attended in the Sperm Analysis Section of São Camilo Laboratory, Maringá (Paraná, Brazil). Eligible men were 18 years or older and had semen analysis requested by their physician as part of a fertility evaluation, after failing to conceive with their partner after one year of unprotected intercourse. Participants who met the following criteria were excluded: symptoms of genitourinary infections, antibiotic treatment within the previous three months, reproductive system abnormalities (e.g., a varicocele), a history of vasectomy, and infertility therapy in the preceding year. Additionally, subjects who tested positive for Chlamydia trachomatis, Ureaplasma urealyticum, Mycoplasma hominis, Mycoplasma genitalium, and Neisseria gonorrhoeae by multiplex polymerase chain reaction assay performed by us [19] were excluded (Figure 1). Participation was voluntary and participants were properly informed about the aim of the study. At recruitment, written informed consent was obtained from all study subjects, who were enrolled only after signing the informed consent form. The study was approved by the Committee for Ethics in Research Involving Humans at the State University of Maringá (UEM)/Paraná, Brazil (permission \#1163409/2015).

\subsection{Laboratory Methods}

2.2.1. Semen Samples. Prior to semen collection for analysis, men were asked to abstain from sexual intercourse or masturbation for 3-5 days. Before semen collection, subjects were instructed to wash their hands with soap, and all samples were collected after urinating and washing the glans penis and coronal sulcus with soap and water. Semen was collected by masturbation and ejaculation directly into standard sterile containers that had previously been shown not to have any cytotoxic effects on human spermatozoa. Participants avoided contact with the interior wall of the sterile container to prevent sample contamination. Freshly collected semen was immediately incubated at $37^{\circ} \mathrm{C}$ for $15-60 \mathrm{~min}$ for liquefaction. Afterwards, samples were homogenized and $300 \mu \mathrm{L}$ was transferred to tubes containing $1.0 \mathrm{~mL}$ of sterile $0.9 \% \mathrm{NaCl}$ solution and immediately stored at $-80^{\circ} \mathrm{C}$ until genomic DNA could be extracted.

2.2.2. Semen Analysis. Detection of basic seminal parameters was performed according to criteria published by the World Health Organization [20]. The following variables were measured: seminal volume, $\mathrm{pH}$, viscosity, sperm concentration, vitality, progressive motility (category $[\mathrm{a}+\mathrm{b}]$ ), morphology (normal forms), leukocytospermia, and hematospermia. Lower reference limits for normal semen characteristics are $\geq 7.2$ for $\mathrm{pH}$ of semen; $1.5 \mathrm{~mL}$ for seminal volume; $15 \times 10^{6} / \mathrm{mL}$ for sperm concentration; 32\% for progressive motility (PR, $\%) ; 58 \%$ for sperm vitality (live spermatozoa, $\%$ ); $4 \%$ for sperm morphology (normal forms, \%); and $<1.0$ for leukocytes. The terms used to describe samples with values lying outside the reference range were hypospermia for seminal 
TABLE 1: Presence of HPV in total semen samples $(n=229)$.

\begin{tabular}{lcc}
\hline HPV presence in semen & $n$ & Percentage (\%) \\
\hline HPV DNA & $38 / 229$ & 16.6 \\
HPV single infection & $24 / 229$ & 10.5 \\
HPV multiple infection & $14 / 229$ & 6.1 \\
Exclusively high-risk HPV genotype(s) & $13 / 229$ & 5.7 \\
Exclusively low-risk HPV genotype(s) & $14 / 229$ & 6.1 \\
\hline
\end{tabular}

Percentage not sum to total due to rounding; HPV: human papillomavirus; DNA: deoxyribonucleic acid.

volume; oligozoospermia for sperm concentration; asthenozoospermia for progressive motility; necrozoospermia for sperm vitality; teratozoospermia for sperm morphology; and leukocytospermia [20].

2.2.3. HPV Detection and Genotyping. To remove any polymerase chain reaction (PCR) inhibitors from the semen, samples were incubated for $15 \mathrm{~min}$ with proteinase $\mathrm{K}$ in phosphate buffered saline and then centrifuged. DNA was extracted using an AxyPrep ${ }^{\mathrm{TM}}$ Body Fluid Viral DNA/RNA Miniprep Kit (Axygen, CA, USA) according to the manufacturer's instructions. The quality and quantity of purified DNA were determined by spectrophotometry on a NanoDrop 2000 Spectrophotometer (Thermo Scientific, Wilmington, USA). HPV was detected by PCR using the primers MY09 and MY11 [21]. This reaction produced a final amplified product of 450 base pairs (bp). The amplification reaction was performed as described by Gimenes et al. [19]. The quality of the DNA was tested by amplification of a 268 bp gene fragment of the human $\beta$-globin gene using the primers GH20 and PC04. Two types of controls were used in the reaction: a sample without DNA (negative control) and an HPV-positive sample (positive control). Final amplified products were loaded onto a $1.0 \%$ agarose gel stained with $150 \mathrm{ng} / \mu \mathrm{L}$ ethidium bromide and subjected to electrophoresis in a horizontal tank at $110 \mathrm{~V}$ for $45 \mathrm{~min}$ in $0.5 \mathrm{x}$ TBE buffer ( $45 \mathrm{mM}$ Tris-borate, $1 \mathrm{mM}$ EDTA, pH 8.0). A 100 bp marker (Invitrogen, Carlsbad, CA, USA) was used as a size standard. The amplified DNA fragments were visualized on a transilluminator with UV light and then photographed. HPV was genotyped by PCRRestriction Fragment Length Polymorphism (PCR-RFLP) analysis, in which amplified DNA was cleaved with restriction enzymes to generate DNA fragments of different molecular sizes. Aliquots of each amplified product were subjected to digestion with the restriction enzyme HpyCH4V (New England Biolabs, Ipswich, MA, USA) as described by Santiago et al. [22]. To better differentiate among HPV genotypes that have similar RFLP patterns, such as HPV 11/30, 18/68, 44/55, and 61/83/84, a second enzyme, NlaIII (New England Biolabs, Ipswich, MA, USA), was used as described by Chen et al. [23]. The restriction digest fragments were subjected to electrophoretic analysis on $8 \%$ polyacrylamide gels. Both 100 and 25 bp ladders (Invitrogen, Carlsbad, CA, USA) were used as molecular size standards. After electrophoresis, polyacrylamide gels were analyzed using LabImage ID software (Loccus Biotechnology, Cotia, SP, Brazil), and the size of each fragment was determined. Genotyping was performed by comparing the molecular weights of fragments for each
HPV genotype as described by Santiago et al. [22]. A total of 39 individual HPV genotypes can be determined by the PCRRFLP method: 17 genotypes are considered to be either $H R$ or potentially $\operatorname{HR}(16,18,31,33,35,39,45,51,52,53,56,58,59$, $66,68,73$, and 82); 22 LR genotypes are not associated with carcinogenesis $(6,11,30,34,40,42,43,44,54,55,61,62,64$, $67,69,70,72,74,81,83,84$, and 91); and the carcinogenic risk for one genotype has not yet been determined (26) [22-25].

2.2.4. Statistical Analysis. Seminal parameters were compared between HPV-positive and HPV-negative groups, HR HPV-positive and HR HPV-negative groups, LR HPVpositive and LR HPV-negative groups, and multiply infected HPV-positive and multiply infected HPV-negative groups using Chi-square tests. The means of age and seminal parameters were compared in the total study population and in strata of HPV presence as follows: HPV-negative; mutually exclusive HPV-positive subgroups (HPV DNA-positive, exclusively HR HPV-positive, exclusively LR HPV-positive, and HPV-positive for multiple infections) by one-way analysis of variance (ANOVA). Two-sided $p$ values $<0.05$ were considered statistically significant. Statistical analysis was performed using GraphPad Prism 6.0 (San Diego, CA, USA).

\section{Results}

After written consent, a total of 229 participants who passed the inclusion and exclusion criteria of the study provided one semen sample each (Figure 1). Participants' mean age was $32.87 \pm 6.6$ years (range 18-52).

The HPV infection rates in semen samples are summarized in Table 1. Overall, 16.6\% (38/229) of the total study population was HPV-positive. Single HPV infections occurred in $10.5 \%(24 / 229)$ of the samples and in $63.2 \%(24 / 38)$ of HPV-positive semen samples. Infections with multiple types of HPV occurred in $6.1 \%$ (14/229) of the samples and in $36.8 \%$ (14/38) of HPV-positive semen samples. In $64.3 \%(9 / 14)$ of samples with multiple HPV types, both LR and HR HPV types were detected simultaneously. HR HPV was detected in the majority of semen samples positive for HPV multiple infections $(13 / 14 ; 93.0 \%)$. HR HPV types were exclusively present in $5.7 \%(13 / 229)$ of samples in single or multiple infections, while $6.1 \%(14 / 229)$ of the samples contained LR HPV types exclusively. The genotype distribution is shown in Table 2. HR HPV16 was the most prevalent genotype in single and multiple infected samples $(9 / 38 ; 23.7 \%)$, followed by LR HPV61 (6/38; 15.8\%), HR HPV82 and LR HPV 43 (5/38; 13.2\% each), and HR HPV58 and LR HPV72 (4/38; 10.5\% each). 
TABLE 2: Detected HPV genotypes in semen (both single and multiple infections).

\begin{tabular}{|c|c|c|c|}
\hline & HPV genotypes & $n$ & Percentage (\%) \\
\hline \multirow{8}{*}{ High-risk } & 16 & 9 & 23.7 \\
\hline & 82 & 5 & 13.2 \\
\hline & 58 & 4 & 10.5 \\
\hline & 53 & 2 & 5.3 \\
\hline & 56 & 2 & 5.3 \\
\hline & 66 & 2 & 5.3 \\
\hline & 18 & 1 & 2.6 \\
\hline & 31 & 1 & 2.6 \\
\hline \multirow{10}{*}{ Low-risk } & 61 & 6 & 15.8 \\
\hline & 43 & 5 & 13.2 \\
\hline & 72 & 4 & 10.5 \\
\hline & 54 & 2 & 5.3 \\
\hline & 62 & 2 & 5.3 \\
\hline & 84 & 2 & 5.3 \\
\hline & 44 & 1 & 2.6 \\
\hline & 69 & 1 & 2.6 \\
\hline & 81 & 1 & 2.6 \\
\hline & 83 & 1 & 2.6 \\
\hline
\end{tabular}

Total HPV-positive samples, $n=38$; indicated frequencies include presence of types both in single and multiple infections.

Table 3 displays the correlation between HPV-positive semen samples and adverse effects on seminal parameters. HPV DNA-positive samples were strongly associated with abnormal seminal viscosity $(p=0.0005)$, and samples with multiple HPV infections were associated with hypospermia $(p=0.01)$ and abnormal seminal viscosity $(p=0.0002)$. Interestingly, semen samples negative for multiple HPV infections were associated with normal seminal viscosity $(p=0.03)$. Still, samples that were HR HPV-positive had a statistically borderline association with abnormal seminal viscosity $(p=0.057)$.

Table 4 lists the means of participant ages and seminal parameters in the total study population and in strata by HPV presence (HPV-negative samples and mutually exclusive HPV-subgroups). The presence of HPV, HR HPV, or LR HPV (separately or in combination) in semen was not related to the mean age of the participants. Having multiple HPV infections was associated with a higher mean seminal $\mathrm{pH}$ and a higher mean number of leukocytes $(p=0.0003$ and $p<0.0001$, resp.).

\section{Discussion}

To our knowledge, this is the first study in Brazil and Latin America to detect and genotype HPV in a large cohort of male partners of couples seeking fertility evaluation and address the possibility that HPV infection may affect seminal parameters. The results of this study reveal that HPV infection in semen is rather common in male partners of infertile couples. Furthermore, we demonstrated an association between HPV seminal infections, particularly infections involving multiple HPV types, and reduction in seminal volume, abnormal viscosity, and elevation of seminal $\mathrm{pH}$. These changes could potentially play important roles in male subfertility and/or infertility.

The possibility that HPV infection could affect seminal parameters and thus fertility is a highly debated topic in the field of human reproduction $[2,4,26]$. We detected an overall HPV prevalence of $16.6 \%$ of the male partners of couples seeking fertility evaluation, which is in accordance with a recent meta-analysis [10] describing a pooled prevalence of $16 \%$ in fertility clinic attendees and with a recent study [9] showing an HPV prevalence of $14.9 \%$ in the male partners of infertile couples. Other studies in asymptomatic men have reported seminal HPV prevalence ranging from 2 to $38.1 \%$ $[9,10,14,17]$, with $16-26 \%$ of semen donors $[8,27]$ and $3-36 \%$ of fertility clinic attendees testing positive for $\operatorname{HPV}[12,13,15]$.

HPV16 was the most prevalent genotype detected in semen samples infected with both single and multiple types, which is in accordance with other recent studies $[9,10]$. However, other usually frequent types, such as HPV6, HPV11, and HPV45, were not detected in this study. In other recent studies, HPV51, HPV52 [27], HPV45, HPV52, HPV18/59 [15], HPV53, and HPVCP61 [28] were the most prevalent genotypes in semen. These data suggest that the prevalence of HPV genotypes may vary based on geographic area. Additional factors such as sociodemographic characteristics, lifestyle, and sexual behavior of the male participants likely contributed to the differences in HPV genotype prevalence. However, as our study was not based on medical records, we do not have data regarding sociodemographic characteristics of our participants.

The high prevalence of HPV DNA and more specifically HPV16 in the semen of asymptomatic males in our study supports previous evidence that semen functions as a transport medium for HPV from the male genital tract to the 


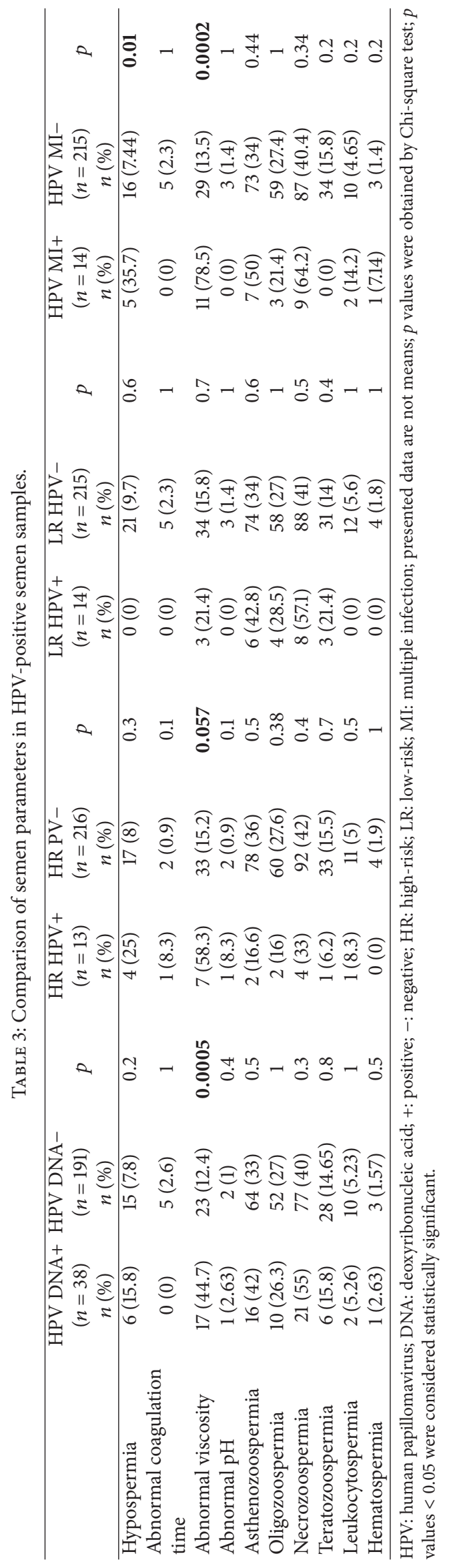




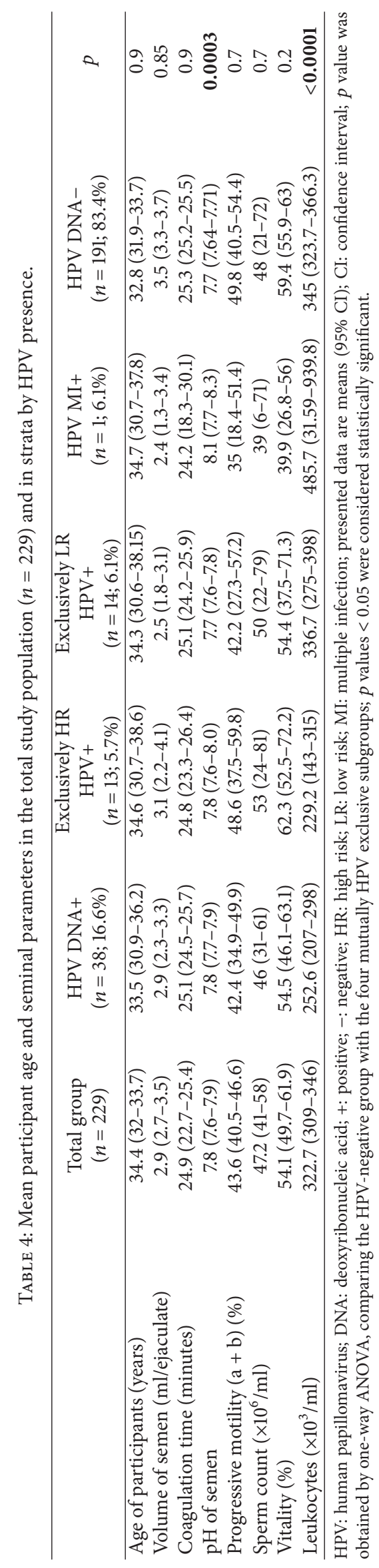


female cervix/uterus [9] and highlights concerns that men with semen infected by HR HPV can potentially be HPV transmitters for a long time. A recent study reported that HPV DNA can be found in semen for 15.3 months and that in males, oncogenic genital HPV infections are more likely to persist for six months or more than nononcogenic infections (61\% and $27 \%$, resp.) [29].

Male fertility depends on the two equally important major components of semen: healthy spermatozoa (in terms of their vitality, motility, and morphology) and the composition of seminal fluid, which is important for sperm function. There was no significant association between HPV infection and sperm concentration, motility, or vitality, as recently described by Luttmer et al. [9]. However, our observations that HPV infection can be associated with reduced semen volume, abnormal viscosity, and increased $\mathrm{pH}$ deserve some consideration.

Secretory products from the seminal vesicles and prostate are crucial for sperm motility, viability, and chromatin stability, but they are also important for semen coagulation and liquefaction. Seminal vesicles secrete the major fraction of the ejaculate $(\approx 60 \%)$ [20]. They produce compounds that play significant roles in seminal physiology, such as fructose, semenogelin-I (the predominant component of the coagulum), and sperm motility stimulators [30-32]. Seminal hyperviscosity suggests deficient secretory activity of the seminal vesicles [33]. Although volume and $\mathrm{pH}$ have less clinical value, they can also yield significant information. The prostate secretes $30-35 \%$ of the ejaculate [20] and produces several compounds that are available for analysis in the seminal plasma, including enzymes for semen liquefaction. Reduced levels of prostate markers are indicative of glandular dysfunction that often associates with abnormalities in $\mathrm{pH}$ ( $\geq 7.8$ ), volume (decreased or increased), liquefaction, and/or viscosity. Our data revealed significant associations between HPV-positive semen samples and hypospermia, abnormal seminal viscosity, higher mean $\mathrm{pH}$, and higher mean number of leukocytes, particularly in semen samples positive for HPV multiple infections. Together, these results indicate that HPVpositive infections in the semen of male partners of couples seeking fertility evaluation may have altered proportions of fluid secreted by the major sexual accessory organs, the prostate, and the seminal vesicles. More specifically, HPV seminal infections appear to lead to changes in prostate markers that are indicative of glandular dysfunction, which may influence fertility. In this context, the association between leukocytospermia and the impairment of prostate markers is remarkable, because it can single out the infection site [34]. Furthermore, as most seminal HPV infections are asymptomatic, leukocytospermia may also be an essential diagnostic probe [35].

Limitations of our study include the absence of data on mixed antiglobulin reaction testing, which was not tested in each primary semen analysis but performed only upon physician's request. Secondly, as our study did not include analysis of medical records, it does not include data on the female partner's characteristics, such as age, hormone levels, tubes, pelvic status, and additional male partner characteristics such as clinical analysis of the prostate and seminal vesicles. Still, semen results were taken from only one semen analysis per subject. Finally, our study did not include normal fertile subjects (control group). However, we believe that our results may encourage further studies to evaluate the influence of $\mathrm{HPV}$ infection on changes in seminal parameters.

\section{Conclusions}

This study is the first to reveal a significant association between HPV-positive semen and hypospermia, abnormal seminal viscosity, higher mean $\mathrm{pH}$, and higher mean numbers of leukocytes, particularly in semen samples positive for multiple HPV infections. Our data support a model where seminal HPV infections lead to changes in prostate markers that are indicative of glandular dysfunction and could change the proportion of fluids secreted from the prostate and the seminal vesicles. Thus, HPV seminal infections could play an important role in male infertility. These results deserve further attention and additional studies should be conducted with larger cohorts.

\section{Conflicts of Interest}

The authors declare that they have no conflicts of interest.

\section{Authors' Contributions}

E. Damke and F. Gimenes were involved in project development, data collection, data analysis, and manuscript writing/editing. E. Damke, F. Kurscheidt, V. Balani, K. I. Takeda, and M. M. T. Irie were involved in data collection and data analysis. M. E. L. Consolaro was involved in project development, data analysis, and manuscript writing/editing.

\section{References}

[1] R. Faridi, A. Zahra, K. Khan, and M. Idrees, "Oncogenic potential of human papillomavirus (HPV) and its relation with cervical cancer," Virology Journal, vol. 8, article no. 269, 2011.

[2] G. Bezold, J. A. Politch, N. B. Kiviat, J. M. Kuypers, H. Wolff, and D. J. Anderson, "Prevalence of sexually transmissible pathogens in semen from asymptomatic male infertility patients with and without leukocytospermia," Fertility and Sterility, vol. 87, no. 5, pp. 1087-1097, 2007.

[3] P. M. Wortley, T. A. Hammett, and P. L. Fleming, "Donor insemination and human immunodeficiency virus transmission," Obstetrics and Gynecology, vol. 91, no. 4, pp. 515-518, 1998.

[4] G. Bayasgalan, D. Naranbat, J. Radnaabazar, T. Lhagvasuren, and P. J. Rowe, "Male infertility: Risk factors in Mongolian men," Asian Journal of Andrology, vol. 6, no. 4, pp. 305-311, 2004.

[5] E. F. Dunne, C. M. Nielson, K. M. Stone, L. E. Markowitz, and A. R. Giuliano, "Prevalence of HPV infection among men: a systematic review of the literature," Journal of Infectious Diseases, vol. 194, no. 8, pp. 1044-1057, 2006.

[6] V. A. Pekler, W. A. Robbins, A. Nyamathi, T. L. Yashina, B. Leak, and T. A. Robins, "Use of Versant ${ }^{\mathrm{TM}}$ TMA and bDNA 3.0 assays to detect and quantify hepatitis C virus in semen," Journal of Clinical Laboratory Analysis, vol. 17, no. 6, pp. 264-270, 2003.

[7] N. Kapranos, E. Petrakou, C. Anastasiadou, and D. Kotronias, "Detection of herpes simplex virus, cytomegalovirus, and Epstein-Barr virus in the semen of men attending an infertility 
clinic," Fertility and Sterility, vol. 79, supplement 3, pp. 15661570, 2003.

[8] M. D. Kaspersen, P. B. Larsen, H. J. Ingerslev et al., "Identification of multiple HPV types on Spermatozoa from human sperm donors," PLoS ONE, vol. 6, no. 3, article e18095, 2011.

[9] R. Luttmer, M. G. Dijkstra, P. J. F. Snijders et al., "Presence of human papillomavirus in semen in relation to semen quality," Human Reproduction, vol. 31, no. 2, pp. 280-286, 2015.

[10] C. Laprise, H. Trottier, P. Monnier, F. Coutlée, and M.-H. Mayrand, "Prevalence of human papillomaviruses in semen: a systematic review and meta-analysis," Human Reproduction, vol. 29, no. 4, pp. 640-651, 2014.

[11] Y. M. Lai, Y. K. Soong, J. F. Lee, F.-P. Yang, H. Y. Huang, and C. C. Pao, "The effect of human papillomavirus infection on sperm cell motility," Fertility and Sterility, vol. 67, no. 6, pp. 1152-1155, 1997.

[12] M. A. M. Rintala, S. E. Grénman, P. P. Pöllänen, J. J. O. Suominen, and S. M. Syrjänen, "Detection of high-risk HPV DNA in semen and its association with the quality of semen," International Journal of STD and AIDS, vol. 15, no. 11, pp. 740743, 2004.

[13] C. Foresta, A. Garolla, D. Zuccarello et al., "Human papillomavirus found in sperm head of young adult males affects the progressive motility," Fertility and Sterility, vol. 93, no. 3, pp. 802-806, 2010.

[14] R. Schillaci, G. Capra, C. Bellavia et al., "Detection of oncogenic human papillomavirus genotypes on spermatozoa from male partners of infertile couples," Fertility and Sterility, vol. 100, no. 5, pp. 1236-1240, 2013.

[15] Y. Yang, C.-W. Jia, Y.-M. Ma, L.-Y. Zhou, and S.-Y. Wang, "Correlation between HPV sperm infection and male infertility," Asian Journal of Andrology, vol. 15, no. 4, pp. 529-532, 2013.

[16] S. Gizzo, B. Ferrari, M. Noventa et al., "Male and couple fertility impairment due to HPV-DNA sperm infection: update on molecular mechanism and clinical impact-systematic review," BioMed Research International, vol. 2014, Article ID 230263, 12 pages, 2014.

[17] F. Gimenes, R. P. Souza, J. C. Bento et al., "Male infertility: a public health issue caused by sexually transmitted pathogens," Nature Reviews Urology, vol. 11, no. 12, pp. 672-687, 2014.

[18] C. Foresta, M. Noventa, L. De Toni, S. Gizzo, and A. Garolla, "HPV-DNA sperm infection and infertility: from a systematic literature review to a possible clinical management proposal," Andrology, vol. 3, no. 2, pp. 163-173, 2015.

[19] F. Gimenes, F. S. Medina, A. L. Abreu et al., "Sensitive simultaneous detection of seven sexually transmitted agents in semen by multiplex-PCR and of HPV by single PCR," PLoS ONE, vol. 9, no. 6, article e98862, 2014.

[20] W. H. Organization, WHO laboratory manual for the examination and processing of human semen, (2010).

[21] M. M. Manos, J. Waldman, T. Y. Zhang et al., "Epidemiology and partial nucleotide sequence of four novel genital human," Journal of Infectious Diseases, vol. 170, no. 5, pp. 1096-1099, 1994.

[22] E. Santiago, L. Camacho, M. L. Junquera, and F. Vázquez, "Full HPV typing by a single restriction enzyme," Journal of Clinical Virology, vol. 37, no. 1, pp. 38-46, 2006.

[23] L. Chen, K. Watanabe, T. Haruyama, and N. Kobayashi, "Simple and rapid human papillomavirus genotyping method by restriction fragment length polymorphism analysis with two restriction enzymes," Journal of Medical Virology, vol. 85, no. 7, pp. 1229-1234, 2013.
[24] V. Bouvard, R. Baan, K. Straif et al., "A review of human carcinogens. Part B: biological agents," The Lancet Oncology, vol. 10, no. 4, pp. 321-322, 2009.

[25] J. Monsonego, J. T. Cox, C. Behrens et al., "Prevalence of highrisk human papilloma virus genotypes and associated risk of cervical precancerous lesions in a large U.S. screening population: data from the ATHENA trial," Gynecologic Oncology, vol. 137, no. 1, pp. 47-54, 2015.

[26] A. Garolla, B. Engl, D. Pizzol et al., "Spontaneous fertility and in vitro fertilization outcome: New evidence of human papillomavirus sperm infection," Fertility and Sterility, vol. 105, no. 1, pp. 65-72, 2016.

[27] A. Perino, L. Giovannelli, R. Schillaci et al., "Human papillomavirus infection in couples undergoing in vitro fertilization procedures: impact on reproductive outcomes," Fertility and Sterility, vol. 95, no. 5, pp. 1845-1848, 2011.

[28] B. Golob, M. Poljak, I. Verdenik, M. Kolbezen Simoniti, E. Vrtačnik Bokal, and B. Zorn, "High HPV infection prevalence in men from infertile couples and lack of relationship between seminal HPV infection and sperm quality," BioMed Research International, vol. 2014, Article ID 956901, 9 pages, 2014.

[29] G. Capra, A. Nyitray, B. Lu, A. Perino, R. Marci, and R. Schillaci, "Analysis of persistence of human papillomavirus infection in men evaluated by sampling multiple genital sites," in European Review for Medical and Pharmacological Sciences, vol. 19, pp. 4153-4163, 2015.

[30] A. Bukovsky, C. J. Thaler, and J. A. McIntyre, "Antigens of immunoglobulin G-Fc receptor III in human male reproductive tract accessory glands," Fertility and Sterility, vol. 55, no. 3, pp. 595-602, 1991.

[31] M. Robert and C. Gagnon, "Sperm motility inhibitor from human seminal plasma: Association with semen coagulum," Molecular Human Reproduction, vol. 1, no. 6, pp. 292-297, 1995.

[32] G. F. Gonzales, G. Kortebani, and A. B. Mazzolli, "Function of seminal vesicles and their role on male fertility," Asian Journal of Information Technology, vol. 3, pp. 251-258, 2001.

[33] G. F. Gonzales, G. Kortebani, and A. B. Mazzolli, "Hyperviscosity and hypofunction of the seminal vesicles," Systems Biology in Reproductive Medicine, vol. 30, no. 1, pp. 63-68, 1993.

[34] F. H. Comhaire, L. Vermeulen, and O. Pieters, "Study of the accuracy of physical and biochemical markers in semen to detect infectious dysfunction of the accessory sex glands.", Journal of Andrology, vol. 10, no. 1, pp. 50-53, 1989.

[35] N. Bar-Chama, E. Goluboff, and H. Fisch, "Infection and pyospermia in male infertility: is it really a problem?" Urologic Clinics of North America, vol. 21, no. 3, pp. 469-475, 1994. 

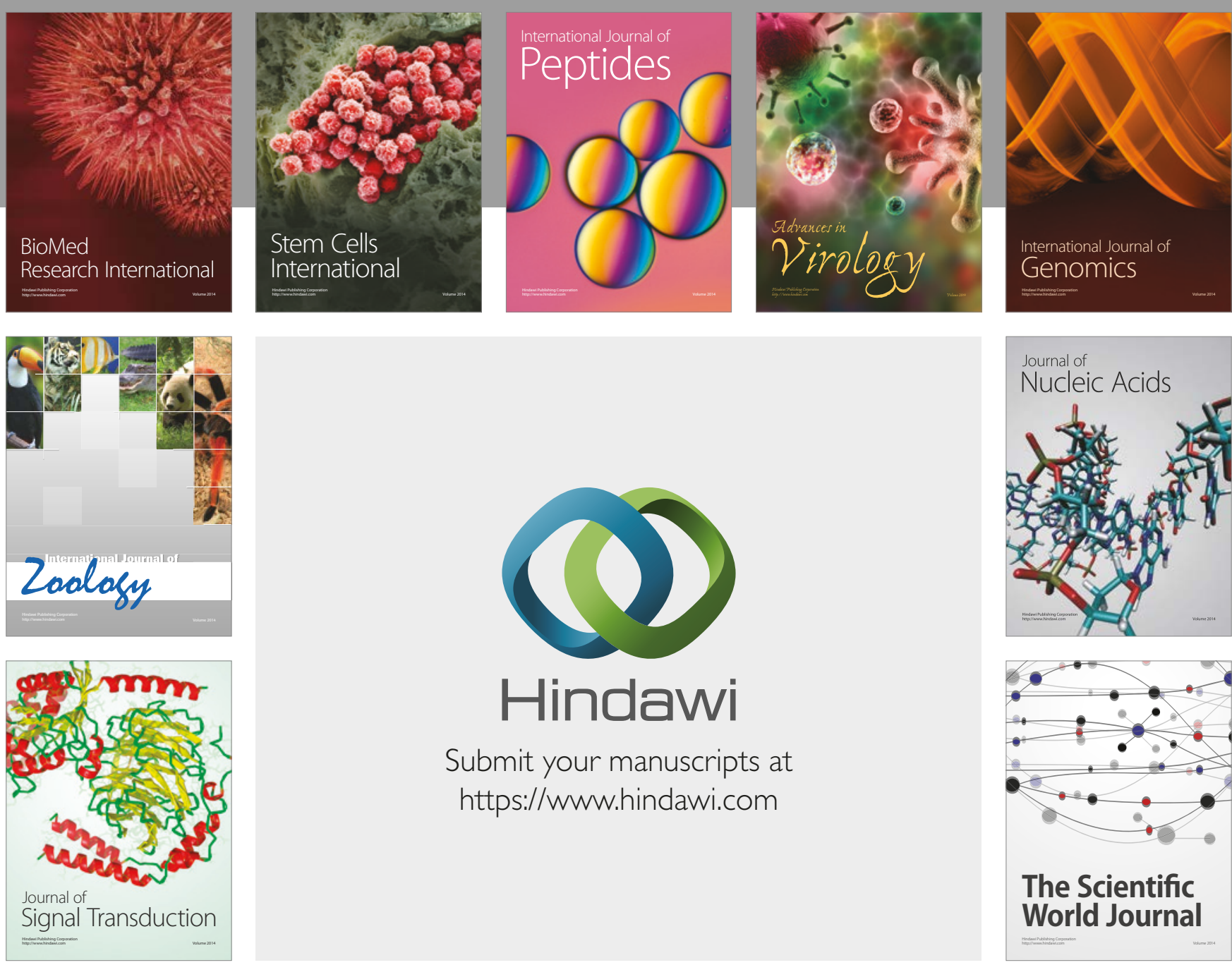

Submit your manuscripts at

https://www.hindawi.com
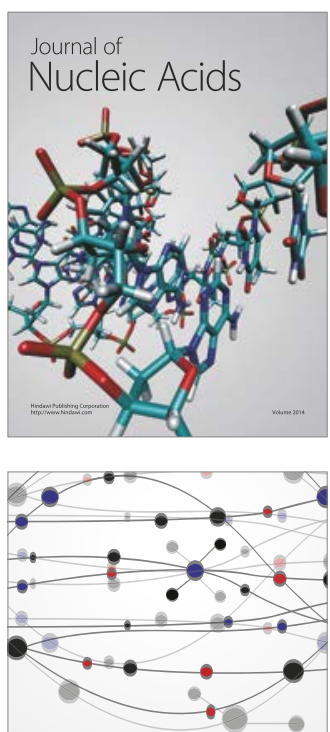

The Scientific World Journal

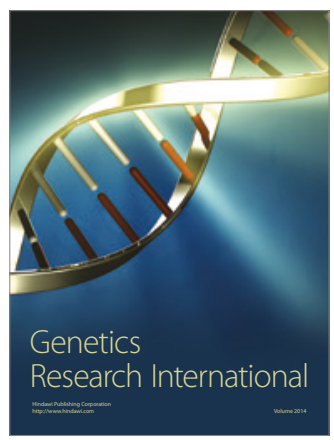

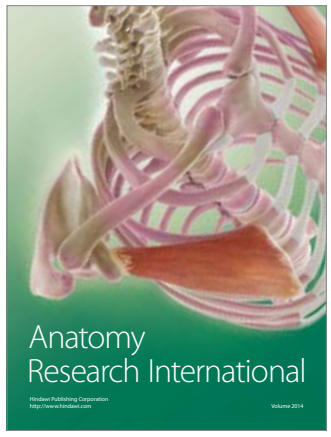

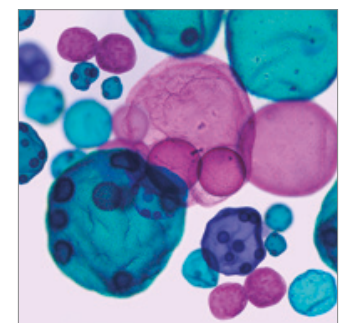

International Journal of Microbiology
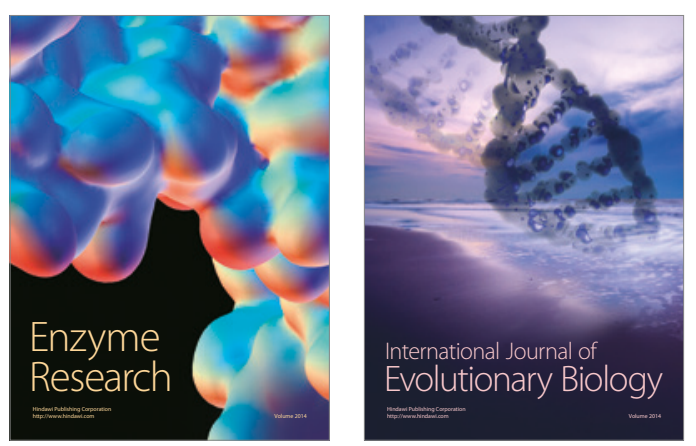
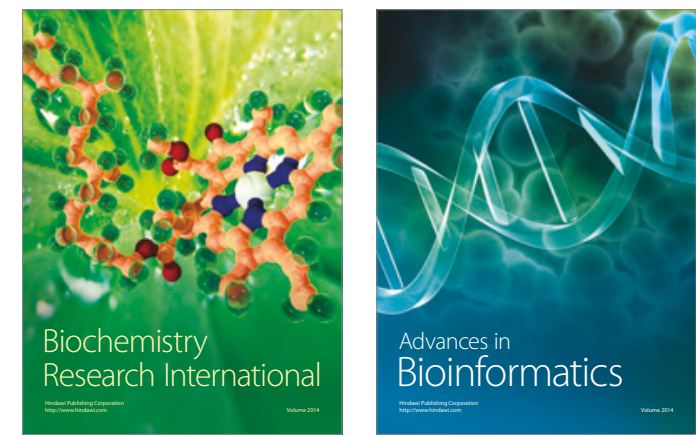

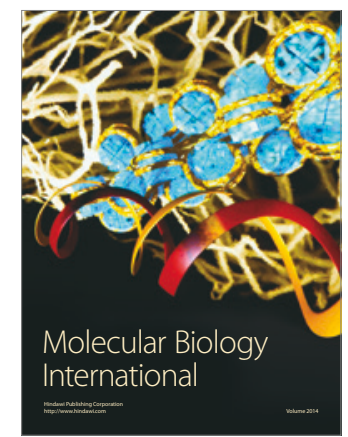

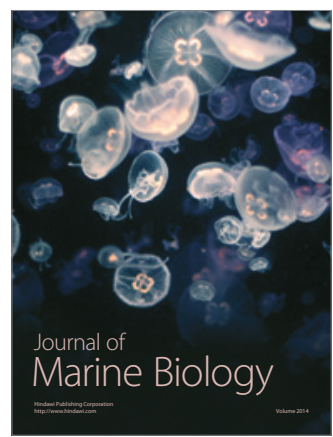

